\title{
Animal cultures matter for conservation
}

\section{Understanding the rich social lives of animals benefits international conservation efforts}

Philippa Brakes, Sasha R. X. Dall, Lucy M. Aplin, Stuart Bearhop, Emma L. Carroll, Paolo Ciucci, Vicki Fishlock, John K. B. Ford, Ellen C. Garland, Sally A. Keith, Peter K. McGregor, Sarah L. Mesnick, Michael J. Noad, Giuseppe Notarbartolo di Sciara, Martha M. Robbins, Mark P. Simmonds, Fernando Spina, Alex Thornton, Paul R. Wade, Martin J. Whiting, James Williams, Luke Rendell, Hal Whitehead, Andrew Whiten, and Christian Rutz

*Corresponding authors. E-mail: p.brakes@exeter.ac.uk; christian.rutz@st-andrews.ac.uk

Animal culture, defined as "information or behavior-shared within a community-which is acquired from conspecifics through some form of social learning" (1), can have important consequences for the survival and reproduction of individuals, social groups, and potentially, entire populations $(1,2)$. Yet, until recently, conservation strategies and policies have focused primarily on broad demographic responses and the preservation of genetically defined, evolutionarily significant units. A burgeoning body of evidence on cultural transmission and other aspects of sociality (3) is now affording critical insights into what should be conserved (going beyond the protection of genetic diversity, to consider adaptive aspects of phenotypic variation), and why specific conservation programs succeed (e.g., through facilitating the resilience of cultural diversity) while others fail (e.g., by neglecting key repositories of socially transmitted knowledge). Here, we highlight how international legal instruments, such as the Convention on the Conservation of Migratory Species of Wild Animals (CMS), can facilitate smart, targeted conservation of a wide range of taxa, by explicitly considering aspects of their sociality and cultures.

\section{CONSEQUENCES OF SOCIAL KNOWLEDGE}

An important aspect of social learning is the speed with which new behaviors can potentially spread through populations, with effects that may be positive (e.g., adaptive exploitation of a new food source) or negative (e.g., increasing conflict with humans, such as when sperm whales learn to remove fish from longlines) (2). Transmission can be mediated by an inherent propensity to adopt innovations (e.g., "lobtail" feeding in humpback whales (1)), or curbed by cultural conservatism (e.g., southern resident killer whales' persistent foraging specialization on Chinook salmon (2)).

Social learning can result in the emergence of subpopulations with distinctive behavioral profiles, erecting social barriers, as observed in distinct vocal clans of sperm whales (see the Figure). Culturally mediated population structure has important implications for conservation efforts (4), as it can influence species-wide phenotypic diversity and adaptability to changing conditions (5). In some cases, such as humpback or blue whale song, cultural variation can reflect demography and facilitate more efficient, or less invasive, assays of contemporary genetic population structure $(1,4)$. Most profoundly, culture can play a causal role in establishing and maintaining distinct evolutionary trajectories (6).

Another consequence of social learning can be the increased importance of key individuals as repositories of accumulated knowledge, making their targeted protection particularly important for the persistence of social units. For example, the experience of African elephant matriarchs (see 
the photo) has been shown to positively influence the fertility rates of younger females in their social group, through the transmission of information about the social and ecological landscape (7). Yet, traditional approaches to species conservation often prioritize younger individuals for their direct reproductive potential.

Positive conservation outcomes can depend on the restoration of cultural knowledge. For example, because whooping cranes learn migratory routes socially, human surrogates in ultralight aircraft can guide naïve, captive-bred birds along their first migration, potentially boosting the effectiveness of reintroduction programs $(8,9)$. Similarly, without the benefit of socially inherited knowledge, bighorn sheep and moose translocated to unfamiliar habitats can take generations to master the skill of tracking the seasonal distribution of high-quality forage (10). Social learning can also be exploited to ameliorate human-wildlife conflict, for example, by artificially "seeding" desirable behavior, such as avoidance of particular foods or sites $(3,11)$.

To improve the efficacy of conservation efforts, we therefore argue that it is critical to consider the interplay between social structure and the transmission of social information. This may be particularly important if different categories of individuals vary in their propensity to innovate, or are more likely to be copied by naïve group members (11). In some cases, populations may be structured into distinct cultural units with differing resource requirements. For instance, cultural transmission of vocal patterns among sperm whales in the Eastern tropical Pacific results in distinct vocal clans (1) (see the Figure). Clans vary in their feeding success during El Niño and La Niña oceanographic cycles (1), meaning that if these cycles increase, as predicted under climate change, population-level impacts may not be uniform.

\section{CETACEANS, AND BEYOND}

Despite mounting evidence that aspects of sociality can have far-reaching implications for wildlife conservation, international policy forums-where most large-scale conservation strategies are conceived-have so far not engaged substantially with the challenges and opportunities presented by this new scientific perspective. A notable exception is the CMS Scientific Council, which has conducted work at the interface of cutting-edge science and international policy-making.

The CMS signatories work to develop collaboration between range states for the conservation of species that move across jurisdictional boundaries. They agree to support research, to endeavor to provide immediate protection for migratory species listed in CMS Appendix I, and to work toward developing agreements for the conservation and management of migratory species in CMS Appendix II. Although, like many other international agreements, CMS does not have a compliance mechanism, its standing committee is in the process of critically reviewing the impacts of its decisions, to improve effectiveness (background CMS documents are available in the Supplementary Materials).

Impetus for an animal culture initiative was provided by a growing body of evidence for social learning and culture in cetaceans that raised important questions about how best to conserve these animals (12). The CMS Scientific Council's pre-existing expertise in evaluating threats to aquatic mammals made CMS a natural avenue for examining these issues further. In 2014, a formal consultation revealed an extensive range of circumstances in which social structure, social learning, and cultural variation in whales and dolphins can affect the planning or outcomes of conservation efforts. This culminated in the adoption of a ground-breaking resolution, through which the CMS signatories formally acknowledged the importance of social learning and culture for the conservation of some highly social species. 
Following the 2014 resolution, the CMS Scientific Council established an expert group to broaden the scope of this initiative beyond cetaceans. The group determined that social learning has conservation relevance across a wide range of vertebrate taxa, including birds, fishes, and many marine and terrestrial mammals $(1,3)$. At a 2018 cross-taxa CMS culture workshop in Parma, Italy, the authors of the present article reviewed relevant evidence, with a particular focus on species in which social learning has the potential to strongly influence migratory behavior, habitat use, foraging, or interaction with human activities. On the basis of this work, we recommend, among other things (for details, see Table S1): augmenting the designation of evolutionarily significant units; conserving individuals that are critical repositories of social knowledge; refining the criteria used for identifying and prioritizing species and populations for assessment; improving reintroduction schemes through strategic management of social knowledge; planning effective mitigation strategies for anthropogenic impacts using aspects of sociality; systematically cataloguing the dimensions of cultural diversity; and raising awareness about the value of conserving animal cultures.

The overall aim of this initiative is to maximize the efficacy of conservation efforts through enhanced consideration of sociality in general, and social learning and (both adaptive, and seemingly arbitrary) cultural processes in particular. Understanding the importance of behavioral diversity will benefit conservation policies both when assessing the status of potentially vulnerable populations (e.g., when delineating units to conserve, by accounting for cultural segregation) and when devising effective conservation strategies (e.g., by identifying key repositories of social knowledge). Achieving these ambitious goals will require a considerable amount of work. For example, although there is broad agreement that successful reintroduction programs require individuals to be behaviorally competent (8), for many species it will still be necessary to establish the degree to which key behaviors are socially learned (e.g., migratory routes in birds $(9,13))$. To facilitate progress, we highlight a few additional opportunities, both in terms of particular species that may merit further consideration and promising research approaches.

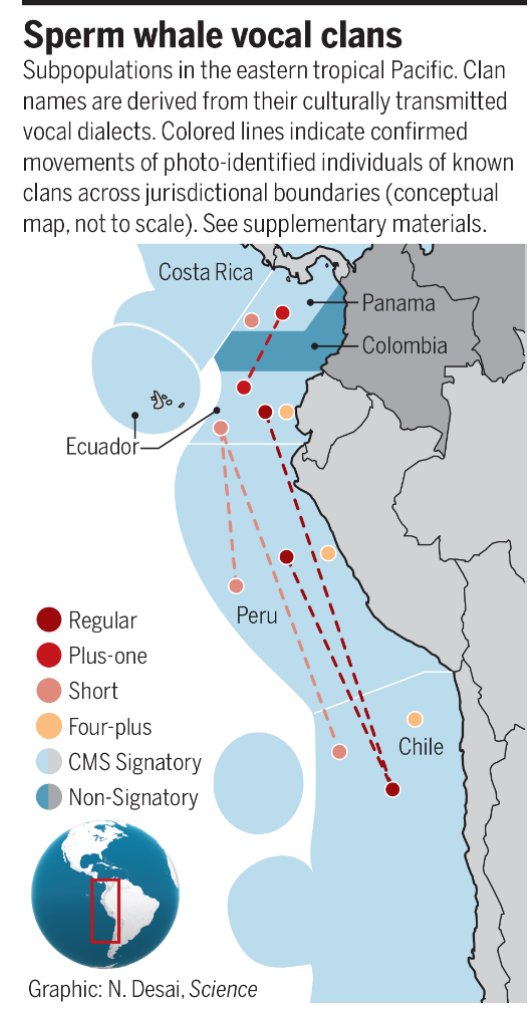


The CMS encourages its signatories to engage in collective conservation measures through its "concerted action" mechanism. This process is particularly relevant when considering collaboration between range states for gathering and sharing data on cultural diversity for populations that move predictably across national borders. In 2017, CMS adopted a concerted action for Eastern tropical Pacific sperm whales (see the Figure). A variety of species may benefit from similar consideration, to evaluate the importance of aspects of their sociality for their conservation. This includes species as diverse as cod (not currently listed in the CMS Appendices), which appear to socially learn migratory routes, and chimpanzees (recently listed in the CMS Appendices), where a culture of nut-cracking tool use thrives in a small area of Western Africa (see Figure S1), yet spans multiple national jurisdictions and may provide access to an important food source during the dry season (3).

An important challenge is to identify those populations, or social units, that would most benefit from our proposed approach, and to predict how specific biological processes may influence conservation outcomes (11). Recent studies illustrate how innovative rapid-assessment techniques could aid the identification of distinct cultural units, which may be particularly vulnerable (e.g., as a result of socially learned foraging strategies). Where socially transmitted traits - such as foraging tactics (and hence resource requirements) and vocal behavior-covary (1), it may be possible to document cultural variation with well-established, inexpensive survey protocols (4). Another approach is to harness new survey technologies, such as autonomous motion-triggered cameras, as exemplified by a recent attempt to chart putative cultural variation in wild chimpanzees (14) in the face of urgent threats from habitat destruction and poaching (see Figure S1). In addition, appropriately parameterized formal models may provide reliable predictions about the impact of specific conservation interventions on sociocultural processes (5). The field of animal social learning is now sufficiently mature to provide key parameters for robust modeling of some systems, where relevant data are available from long-term field studies and controlled experiments.

\section{MOVING FORWARD}

Our growing understanding of the conservation relevance of cultural variation urges that scientists and policy-makers collaborate closely to ensure that policy is informed by the latest scientific advances. Many cultural systems are highly complex, and the conservation impact of cultural processes is context dependent, necessitating careful case-by-case consideration. Recommendations from the Parma workshop will inform discussions at the November 2019 Meeting of the Sessional Committee of the CMS Scientific Council and the 2020 CMS Conference of the Parties in India. A key challenge will be to determine if evidence warrants explicitly recognizing some distinct cultural units listed in the CMS Appendices, and how insights from this work can be used to inform conservation efforts across the entire CMS portfolio of agreements.

Within the broader context of international wildlife law (15), it is important to consider the relevance of animal culture for scientific assessments and policy decision-making across a range of relevant multilateral environmental agreements, such as the Convention on International Trade in Endangered Species of Wild Fauna and Flora (CITES) and the Convention on Biological Diversity (CBD). We see opportunities to extend our approach beyond species and issues currently covered by CMS, for example, when assessing the sustainability of exports and trade through CITES processes. Such consideration is timely, because 2020 is the final year of the United Nations Decade on Biodiversity, when governments will negotiate the Post-2020 Biodiversity Framework. Given the 
prevalence of social learning and cultures across a wide range of taxa, a comprehensive, integrated approach is essential to maintaining the natural diversity and integrity of Earth's rich ecosystems.

\section{REFERENCES AND NOTES}

1. H. Whitehead, L. Rendell, The Cultural Lives of Whales and Dolphins (Univ. of Chicago Press, 2015).

2. H. Whitehead, Learn. Behav. 38, 329 (2010).

3. A. Whiten, Interface Focus 7, 20160142 (2017).

4. E. C. Garland et al., Conserv. Biol. 29, 1198 (2015).

5. S. A. Keith, J. W. Bull, Ecography 40, 296 (2017).

6. A. D. Foote et al., Nat. Commun. 7, 11693 (2016).

7. K. McComb, C. Moss, S. M. Durant, L. Baker, S. Sayialel, Science 292, 491 (2001).

8. T. Mueller, R. B. O'Hara, S. J. Converse, R. P. Urbanek, W. F. Fagan, Science 341, 999 (2013).

9. C. S. Teitelbaum, S. J. Converse, T. Mueller, Conserv. Lett. 12, e12599 (2019).

10. B. R. Jesmer et al., Science 361, 1023 (2018).

11. A. L. Greggor, A. Thornton, N. S. Clayton, Behav. Ecol. Sociobiol. 71, 16 (2017).

12. H. Whitehead, L. Rendell, R. W. Osborne, B. Würsig, Biol. Conserv. 120, 427 (2004).

13. B.-U. Meyburg et al., J. Exp. Biol. 220, 2765 (2017).

14. H. S. Kühl et al., Sci. Rep. 6, 22219 (2016).

15. A. Trouwborst et al., BioScience 67, 784 (2017).

\section{ACKNOWLEDGMENTS}

We are grateful to the CMS executive secretary and the CMS secretariat for organizing the Parma workshop; the hosts and sponsors of the workshop (the Appennino Tosco-Emiliano National Park, the Fondazione Monteparma, and the Principality of Monaco); M. Prideaux for helpful policy discussions; S. Smart for graphic design; and the experts that have supported this initiative. Competing interests: G.N.d.S. is the CMS Conference of the Parties Appointed Councillor for Aquatic Mammals. F.S. is the chair of the CMS Scientific Council and is the councillor for Italy. J.W. is the UK scientific councillor for CMS and works for the Joint Nature Conservation Committee, which is a statutory adviser to the UK Government.

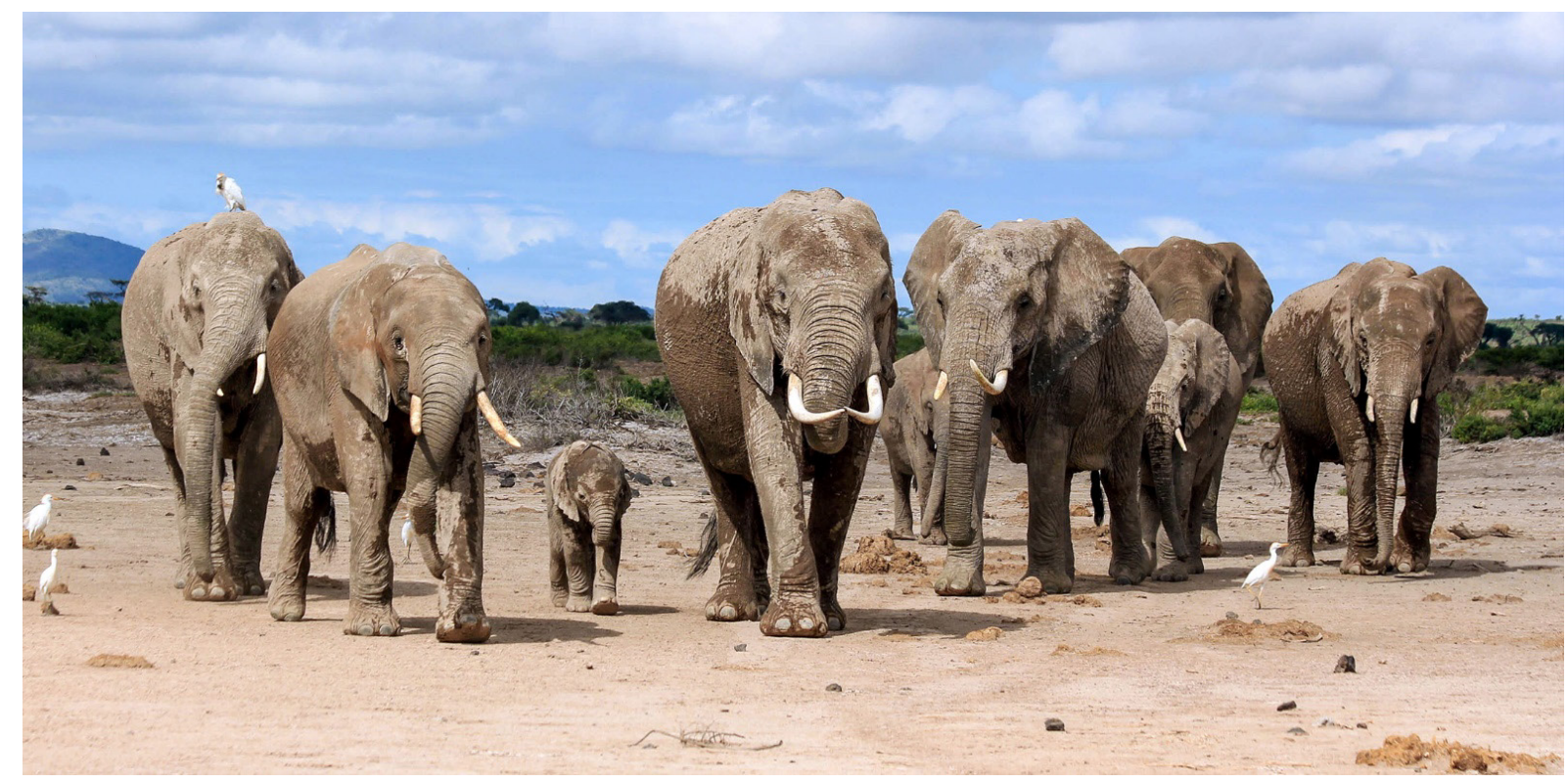


Supplementary Materials 


\section{Author Affiliations}

${ }^{1}$ Centre for Ecology and Conservation, Biosciences, College of Life and Environmental

Sciences, University of Exeter, Penryn, Cornwall TR10 9FE, UK.

${ }^{2}$ Whale and Dolphin Conservation, Brookfield House, 38 St Paul Street, Chippenham, Wiltshire SN15 1LJ, UK.

${ }^{3}$ Max Planck Institute for Ornithology, Radolfzell, 78315, Germany.

${ }^{4}$ Edward Grey Institute, Department of Zoology, University of Oxford, Oxford OX1 3PS, UK.

${ }^{5}$ School of Biological Sciences, University of Auckland, 3A Symonds Street, Auckland 1010,

New Zealand.

${ }^{6}$ Sea Mammal Research Unit, School of Biology, University of St Andrews, St Andrews KY16 8LB, UK.

${ }^{7}$ Department of Biology and Biotechnologies, University of Rome La Sapienza, 00185, Roma, Italy.

${ }^{8}$ Amboseli Trust for Elephants, PO Box 15135, Langata 00509, Nairobi, Kenya.

${ }^{9}$ School of Natural Sciences, University of Stirling, Stirling FK9 4LA, UK.

${ }^{10}$ Department of Zoology, University of British Columbia, Vancouver BC, Canada.

${ }^{11}$ Centre for Social Learning and Cognitive Evolution, School of Biology, University of St Andrews, St Andrews KY16 9TH, UK.

${ }^{12}$ Lancaster Environment Centre, Lancaster University, Lancaster LA1 4YQ, UK.

${ }^{13}$ ISPA - Instituto Universitário, Rua Jardim do Tabaco 34, 1149-041 Lisboa, Portugal.

${ }^{14}$ Centre for Applied Zoology, Cornwall College Newquay, Cornwall TR7 2LZ, UK.

${ }^{15}$ Marine Mammal and Turtle Division, Southwest Fisheries Science Center, National Marine

Fisheries Service, National Oceanic and Atmospheric Administration, 8901 La Jolla Shores

Drive, La Jolla, CA 92037, USA.

${ }^{16}$ Scripps Institution of Oceanography, UC San Diego, 9500 Gilman Drive, La Jolla, CA 920930203, USA.

${ }^{17}$ Cetacean Ecology and Acoustics Laboratory, School of Veterinary Science, The University of Queensland, Qld 4343, Australia.

${ }^{18}$ Tethys Research Institute, Viale G.B. Gadio 2, 20121 Milano, Italy.

${ }^{19}$ Department of Primatology, Max Planck Institute for Evolutionary Anthropology, Deutscher Platz 6, Leipzig, Germany.

${ }^{20}$ Bristol Veterinary School, University of Bristol, Langford House, Langford, Bristol BS40 5DU, UK.

${ }^{21}$ Humane Society International, c/o 5 Underwood Street, Hoxton, London N1 7LY, UK.

${ }^{22}$ Istituto Superiore Protezione Ricerca Ambientale (ISPRA), Via Cà Fornacetta 9, I-40064

Ozzano Emilia (BO), Italy.

${ }^{23}$ Marine Mammal Laboratory, Alaska Fisheries Science Center, NOAA Fisheries, Seattle, WA 98177, USA.

${ }^{24}$ School of Aquatic and Fishery Sciences, University of Washington, 1122 NE Boat Street, Seattle, WA 98105, USA.

${ }^{25}$ Department of Biological Sciences, Macquarie University, Sydney, NSW 2109, Australia.

${ }^{26}$ Joint Nature Conservation Committee, Monkstone House, City Road, Peterborough PE1 1JY, UK.

${ }^{27}$ Biology Department, Dalhousie University, Halifax, Nova Scotia, B3H4R2, Canada.

${ }^{28}$ Centre for Social Learning and Cognitive Evolution, School of Psychology and Neuroscience, 
University of St Andrews, St Andrews KY16 9JP, UK.

${ }^{29}$ Centre for Biological Diversity, School of Biology, University of St Andrews, St Andrews KY16 9TH, UK.

\section{Materials}

Sperm whale graphic

The graphic shows the ranges of distinct sub-populations of sperm whales (Physeter macrocephalus) in the Eastern tropical Pacific, with names derived from the culturallytransmitted vocal dialects ('regular', 'plus-one', etc.; colored circles) used to identify them in the field [data from $(16,17)$; see Table S2]. New findings show that sperm whale population structure is strongly defined by membership of these dialect groups, termed 'vocal clans'. For some clans, movements of photo-identified individuals across jurisdictional boundaries have been confirmed [colored dashed lines; data from $(17,18)$; see Table S3]. Clan ranges not only span multiple national jurisdictions, but they also structure populations within jurisdictions in ways that are difficult or impossible to detect without data on cultural variation. Since clans appear to vary in how they respond to environmental change (19), this information has clear policy implications: $(i)$ signatories of the Convention on the Conservation of Migratory Species of Wild Animals (CMS) should cooperate on management plans for clans that range between jurisdictions; and (ii) conservation efforts may need to be tailored to emerging knowledge about behavioral variation between clans. Sperm whales are listed on CMS Appendices I and II. The graphic is a conceptual representation only - the delineation and size of exclusive economic zones (EEZs), placement of vocal clans, and movements of individuals have been adjusted for illustrative purposes.

CMS documents relating to statements in the main text

- UNEP/CMS/COP11/Inf.18: Report of the CMS Scientific Council Workshop on the Conservation Implications of Cetacean Culture

https://www.cms.int/en/document/report-cms-scientific-council-workshop-conservationimplications-cetacean-culture-0

- UNEP/CMS/Resolution 11.23: Conservation Implications of Cetacean Culture https://www.cms.int/en/document/conservation-implications-cetacean-culture-1

- UNEP/CMS/ScC-SC3/Inf.8: Report of the CMS Workshop on Conservation Implications of Animal Culture and Social Complexity https://www.cms.int/en/document/report-cms-workshop-conservation-implications-animalculture-and-social-complexity

- UNEP/CMS/StC48/Report: Report of the 48th Meeting of the Standing Committee https://www.cms.int/sites/default/files/document/cms_stc48 report e.pdf

- UNEP/CMS/COP12/Doc.21.1.32: Resolutions to repeal in part Resolution 11.23, Conservation Implications of Cetacean Culture https://www.cms.int/sites/default/files/document/cms_cop12_doc.21.1.32 res 11.23 cetacea n-culture e $0 . p d f$

- UNEP/CMS/Concerted Action 12.2: Concerted Action for the Sperm Whales (Physeter macrocephalus) of the Eastern Tropical Pacific https://www.cms.int/en/document/concerted-action-sperm-whales-physeter-macrocephaluseastern-tropical-pacific 
$\underline{\text { Parma declaration }}$

Statement from CMS Workshop on Conservation Implications of Animal Culture and Social Complexity, held in Parma, Italy, 12-14 April 2018:

"A group of international experts in behavioural ecology and conservation biology met for a workshop under the auspice of the Scientific Council of the United Nations Convention on Migratory Species, in Parma, Italy, to examine the implications of non-human (hereafter 'animal') culture and sociality for conservation efforts. The workshop was kindly supported by the Appennino Tosco-Emiliano National Park and Fondazione Monteparma and the Government of Monaco under the Migratory Species Champion Programme.

The Parma workshop participants explored and acknowledged that there is now an impressive body of scientific evidence for culture and transmission of social knowledge across a wide range of vertebrate and invertebrate taxa (including cetaceans, great apes, elephants, other mammals, birds, fish and some reptiles). In some cases this gives rise to a number of challenges - and new opportunities - associated with conserving these social species.

The UN Convention on Migratory Species, which spearheaded this initiative, is the first multilateral environmental agreement to tackle the issue of social learning and culture and its significance for conservation outcomes. These issues have been discussed at the highest level of the Convention - by the Conference of the Parties to the treaty - where the Parties have agreed and formally endorsed this initiative, acknowledging the importance of considering these emergent aspects of conservation management efforts.

Evidence based evaluation of social learning and resultant non-human culture (hereafter 'culture') indicates that these processes may be important for population trends across a wide range of vertebrate taxa. The social transmission of knowledge between individuals, and culture, may increase social group and population viability and provide opportunities for the rapid spread of innovations and thus adaptation to environmental change. It can also act as a proxy for identification of population structure which is important for conservation.

Burgeoning threats to habitats and species, from climate change and other anthropogenic influences, necessitate that conservation efforts are as streamlined and efficient as possible. The strategic use of knowledge regarding social learning processes may be an important tool to facilitate restoration across a broad range of migratory taxa. For example, in helping released birds re-learn historical migratory routes.

Resource requirements and management of social units may differ across cultures within the same species, for example across cultures characterised by very different foraging strategies. Thus the conservation and management of these social units may need to be adapted according to their resource needs.

Where social information is important to the survival of a social group, and specifically where the social group relies upon individuals, classes of individuals or groups that act as repositories of social knowledge, the removal of individuals from populations of socially complex species may have consequences beyond simply a reduction in absolute numbers and repercussions for the transmission of foraging traditions, migratory routes and other behaviours critical for populations to thrive: individuals matter. More broadly this has implications for preserving age structure in populations where possible.

Whilst there are many challenges associated with identifying repositories of social knowledge and protecting social capital within a social unit, it was agreed that some 
populations may be best delineated by cultural behaviour, rather than just differentiated by genetic diversity or geographic isolation.

Thus, concerned that highly social species face unique conservation challenges, the participants at the workshop advocate a precautionary and practical approach for the management of populations for which there is scientific evidence that the influence of culture, demography, social network structure and connectivity should be considered along with other aspects of conservation for that species."

\section{Supplementary Text}

\section{Acknowledgments}

We are grateful to: the CMS Executive Secretary and the CMS Secretariat for organizing the Parma workshop; the hosts and sponsors of the workshop (the Appennino Tosco-Emiliano National Park, the Fondazione Monteparma, and the Principality of Monaco); Margi Prideaux for helpful policy discussions; Steve Smart for graphic design; the large number of experts that have supported this initiative over the years; and four anonymous reviewers and the editor for excellent comments that improved the paper.

\section{Author contributions}

All co-authors attended the CMS Workshop on Conservation Implications of Animal Culture and Social Complexity, 12-14 April 2018, Parma, Italy, except LR, who had contributed to launching this initiative in 2014. PB and CR co-wrote the manuscript, with contributions from SRXD, LR, AW and HW. PB and CR developed ideas for the figures. All co-authors contributed to the development of ideas and provided feedback on the manuscript.

\section{Competing interests}

GNdS declares that he is the CMS Conference of the Parties Appointed Councillor for Aquatic Mammals. FS declares that he is the Chair of the CMS Scientific Council, and Councillor for Italy. JW declares that he is the UK Scientific Councillor for CMS, and that the Joint Nature Conservation Committee is a statutory advisor to the UK Government. The other co-authors declare that they have no competing interests. 


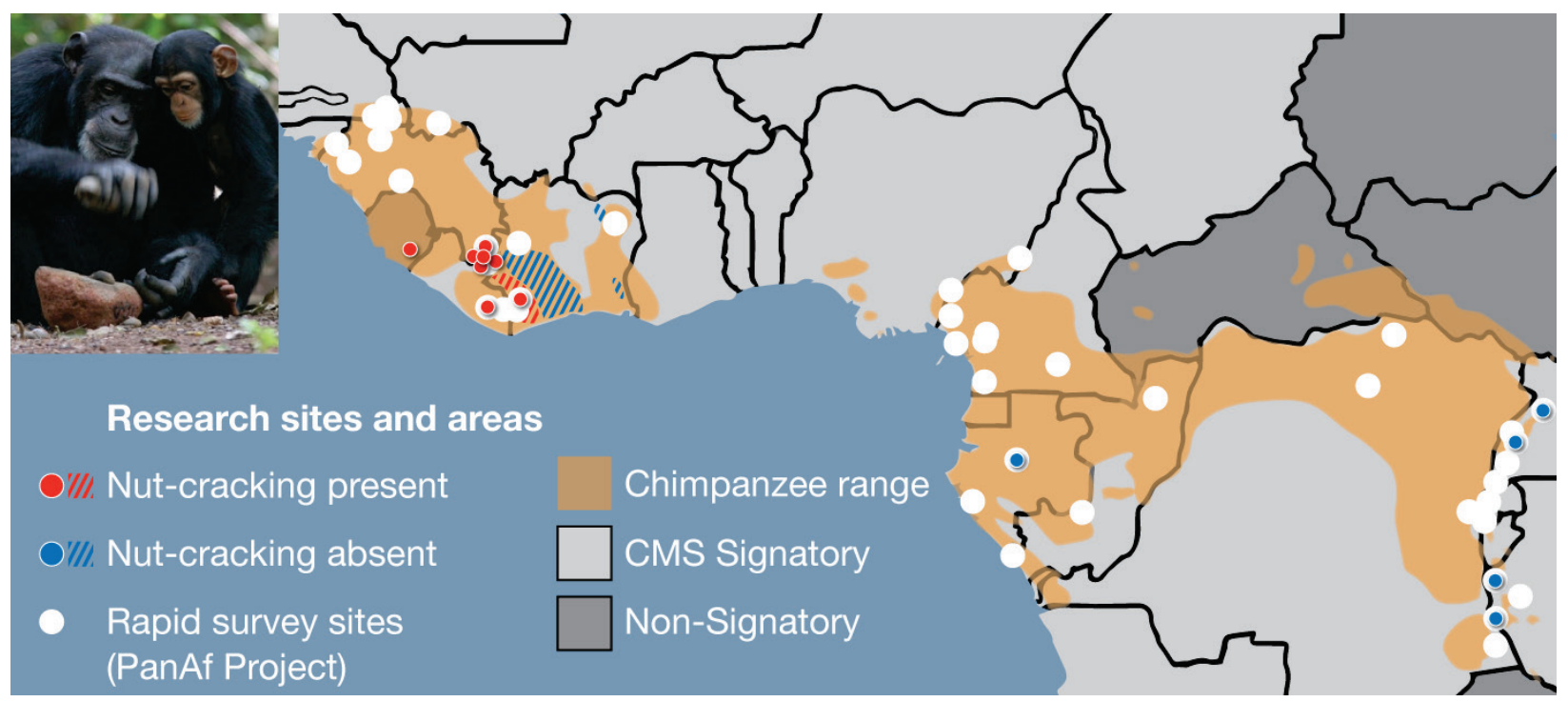

Fig. S1. The graphic shows the distribution of the chimpanzee (Pan troglodytes) across Africa [orange shading; ranges of four sub-species combined, adapted from (14)], as well as the location of selected long-term research sites [colored circles; data from $(20,21)]$, larger survey areas [colored hatching; adapted from (22)], and rapid-assessment survey sites of the 'Pan African Programme: The Cultured Chimpanzee' (PanAf) [white circles; data from (14)]. Previous research suggested that the presence (red circles and hatching) and apparent absence (blue circles and hatching) of nut-cracking behavior reflects cultural differences, with the large Sassandra River in Côte d'Ivoire acting as a transmission barrier [for detailed discussion, see (3)]. Nut cracking has been reported from the Ebo Forest of Cameroon (23), but since its occurrence and distribution remain to be investigated systematically, the site is not included here. The PanAf project is a field study of unprecedented scale, and has the potential to uncover previouslyunknown cultural variation, with important implications for conservation efforts. While chimpanzees do not exhibit seasonal migrations, the species is considered under the CMS, as dispersal takes place across jurisdictional boundaries, and it has recently been listed on CMS Appendices I and II. Photo: Primate Research Institute, Kyoto University. 
Table S1. Extended summary recommendations from the CMS Workshop on Conservation Implications of Animal Culture and Social Complexity in 2018 in Parma, Italy.

Issue

Augment designation of evolutionarily significant units (ESUs) to include cultural variation

Integrate cultural repositories and capacities into management

Educate and raise awareness

Collect empirical evidence

Expand theoretical modelling

Catalogue dimensions of cultural diversity

\section{Workshop recommendations}

Culture should, when appropriate, be incorporated into the designation of population units meriting attention for conservation efforts, complementing the traditional focus on species, and on genetics.

Conserving cultural repositories and capacities (e.g., cultural inheritance systems) should be integrated into the development of IUCN, CMS and other conservation and management strategies; including, but not limited to, assessing populations and designating units to conserve, in situ monitoring, and managing human-wildlife conflict and reintroduction programs.

Raising awareness and providing education on the value of conserving cultural diversity should be a priority of the CMS initiative on culture and social complexity in animals.

Empirical evidence of behavioral diversity, social learning networks, and migratory behavior should be collected for taxa of relevance to CMS.

Theoretical models of social transmission and population-level effects should be developed, to inform mitigation efforts and investigate future scenarios for conservation issues.

Cataloguing the dimensions of cultural diversity in animals may be important:

a) to identify and conserve cultural capacities and repositories;

b) to inform conservation actions and strategies. 


\section{Additional recommendations}

Innovate methods for rapid assessment

\section{Develop assessment criteria}

Broaden taxonomic scope
Innovative rapid-assessment techniques can aid the effective identification of distinct social/cultural units, and it is critical:

a) to examine opportunities to use selected traits of co-varying cultural variants as 'markers';

b) to explore novel survey technologies, e.g., large-scale deployment of autonomous motion-triggered cameras;

c) to consider empirically parameterized computer simulations for providing reliable predictions about the effects of social processes, or the impact of specific conservation interventions.

Develop further the criteria to identify species for which aspects of sociality might be important to their conservation, to help practitioners understand when cultural transmission might impact their decision making.

Broaden the taxonomic scope of this initiative to elucidate other taxa and social processes relevant to conserving social species. 
Table S2. CMS range states in which different vocal clans of sperm whales have been found; data were extracted from two published sources [X from (16), and Y from (17)]. In the graphic in the main text, results for Galápagos and mainland Ecuador are combined.

\begin{tabular}{lccccc}
\hline \hline Clan & Panama & Galápagos & $\begin{array}{c}\text { Ecuador } \\
\text { (mainland) }\end{array}$ & Peru & Chile \\
\hline Regular & $\mathrm{X}$ & $\mathrm{X}$ & $\mathrm{X}$ & $\mathrm{X}$ & $\mathrm{X}$ \\
Plus-one & $\mathrm{X}$ & $\mathrm{X}, \mathrm{Y}$ & $\mathrm{X}$ & $\mathrm{X}$ & $\mathrm{X}$ \\
Short & & $\mathrm{Y}$ & & $\mathrm{X}$ & $\mathrm{X}$ \\
Four-plus & & & & \\
\hline \hline
\end{tabular}


Table S3. Movements of sperm whale individuals of known vocal clans between CMS range states, using photo-identification data from (18) and acoustic data from (17).

\begin{tabular}{lcccc}
\hline \hline & Panama & Ecuador & Peru & Chile \\
\hline Panama & - & - & - & - \\
Ecuador & 8 Plus-one & - & - & - \\
Peru & & 2 Short & - & - \\
Chile & 1 Regular; 5 Short & 1 Regular & \\
\hline \hline
\end{tabular}




\section{References}

16. L. Rendell, H. Whitehead, Vocal clans in sperm whales (Physeter macrocephalus). Proc. $R$. Soc. B 270, 225-231 (2003).

17. M. Cantor, H. Whitehead, S. Gero, L. Rendell, Cultural turnover among Galápagos sperm whales. R. Soc. Open Sci. 3, 160615 (2016).

18. H. Whitehead, A. Coakes, N. Jaquet, S. Lusseau, Movements of sperm whales in the tropical Pacific. Mar. Ecol. Prog. Ser. 361, 291-300 (2008).

19. H. Whitehead, L. Rendell, Movements, habitat use and feeding success of cultural clans of South Pacific sperm whales. J. Anim. Ecol. 73, 190-196 (2004).

20. A. Whiten, J. Goodall, W. C. McGrew, T. Nishida, V. Reynolds, Y. Sugiyama, C. E. G. Tutin, R. W. Wrangham, C. Boesch, Charting cultural variation in chimpanzees. Behaviour 138, 1481-1516 (2001).

21. S. Carvalho, W. McGrew, The origins of the Oldowan: Why chimpanzees (Pan troglodytes) still are good models for technological evolution in Africa, in Stone Tools and Fossil Bones: Debates in the Archaeology of Human Origins, M. Domínguez-Rodrigo, Ed. (Cambridge University Press, Cambridge, 2012), pp. 201-221.

22. C. Boesch, P. Marchesi, N. Marchesi, B. Fruth, F Joulian. Is nutcracking in wild chimpanzees a cultural behaviour? J. Hum. Evol. 26, 325-338 (1994).

23. B. J. Morgan, E. E. Abwe, Chimpanzees use stone hammers in Cameroon. Curr. Biol. 16, R632-R633 (2006). 